\title{
Functional Dependence in Strategic Games
}

\author{
Kristine Harjes and Pavel Naumov
}

\begin{abstract}
The article studies properties of functional dependencies between strategies of players in Nash equilibria of multiplayer strategic games. The main focus is on the properties of functional dependencies in the context of a fixed dependency graph for payoff functions. A logical system describing properties of functional dependence for any given graph is proposed and is proven to be complete.
\end{abstract}

\section{Introduction}

Functional dependence. In this article we study dependency between players' strategies in Nash equilibria. For example, the coordination game described by Table 1 has two Nash equilibria: $\left(a_{1}, b_{1}\right)$ and $\left(a_{2}, b_{2}\right)$. Knowing the strategy of player $a$ in a Nash equilibrium of this game, one can predict the strategy of player $b$. We say that player $a$ functionally determines player $b$ and denote this by $a \triangleright b$.

Note that, in the case of the coordination game, we also have $b \triangleright a$. However, for the game described by Table 2, the statement $a \triangleright b$ is true, but $b \triangleright a$ is false.

The main focus of this article is functional dependence in multiplayer games. For example, consider a "parity" game with three players $a, b, c$. Each of the players picks 0 or 1, and all players are rewarded if the sum of all three numbers is even. This game has four different Nash equilibria: $(0,0,0),(0,1,1),(1,0,1)$, and $(1,1,0)$. It is easy to see that knowledge of any two players' strategies in a Nash equilibrium

Table 1 Coordination game.

\begin{tabular}{|c|c|c|}
\hline & $b_{1}$ & $b_{2}$ \\
\hline$a_{1}$ & 1,1 & 0,0 \\
\hline$a_{2}$ & 0,0 & 1,1 \\
\hline
\end{tabular}

Received March 20, 2013; accepted October 29, 2013

First published online February 25, 2016

2010 Mathematics Subject Classification: Primary 03B80

Keywords: strategic games, functional dependency, axiomatization, completeness

(C) 2016 by University of Notre Dame 10.1215/00294527-3479096 


\title{
Functional Dependence in Strategic Games
}

\author{
Kristine Harjes and Pavel Naumov
}

\begin{abstract}
The article studies properties of functional dependencies between strategies of players in Nash equilibria of multiplayer strategic games. The main focus is on the properties of functional dependencies in the context of a fixed dependency graph for payoff functions. A logical system describing properties of functional dependence for any given graph is proposed and is proven to be complete.
\end{abstract}

\section{Introduction}

Functional dependence. In this article we study dependency between players' strategies in Nash equilibria. For example, the coordination game described by Table 1 has two Nash equilibria: $\left(a_{1}, b_{1}\right)$ and $\left(a_{2}, b_{2}\right)$. Knowing the strategy of player $a$ in a Nash equilibrium of this game, one can predict the strategy of player $b$. We say that player $a$ functionally determines player $b$ and denote this by $a \triangleright b$.

Note that, in the case of the coordination game, we also have $b \triangleright a$. However, for the game described by Table 2, the statement $a \triangleright b$ is true, but $b \triangleright a$ is false.

The main focus of this article is functional dependence in multiplayer games. For example, consider a "parity" game with three players $a, b, c$. Each of the players picks 0 or 1, and all players are rewarded if the sum of all three numbers is even. This game has four different Nash equilibria: $(0,0,0),(0,1,1),(1,0,1)$, and $(1,1,0)$. It is easy to see that knowledge of any two players' strategies in a Nash equilibrium

Table 1 Coordination game.

\begin{tabular}{|c|c|c|}
\hline & $b_{1}$ & $b_{2}$ \\
\hline$a_{1}$ & 1,1 & 0,0 \\
\hline$a_{2}$ & 0,0 & 1,1 \\
\hline
\end{tabular}

Received March 20, 2013; accepted October 29, 2013

First published online February 25, 2016

2010 Mathematics Subject Classification: Primary 03B80

Keywords: strategic games, functional dependency, axiomatization, completeness

(C) 2016 by University of Notre Dame 10.1215/00294527-3479096 


\title{
Functional Dependence in Strategic Games
}

\author{
Kristine Harjes and Pavel Naumov
}

\begin{abstract}
The article studies properties of functional dependencies between strategies of players in Nash equilibria of multiplayer strategic games. The main focus is on the properties of functional dependencies in the context of a fixed dependency graph for payoff functions. A logical system describing properties of functional dependence for any given graph is proposed and is proven to be complete.
\end{abstract}

\section{Introduction}

Functional dependence. In this article we study dependency between players' strategies in Nash equilibria. For example, the coordination game described by Table 1 has two Nash equilibria: $\left(a_{1}, b_{1}\right)$ and $\left(a_{2}, b_{2}\right)$. Knowing the strategy of player $a$ in a Nash equilibrium of this game, one can predict the strategy of player $b$. We say that player $a$ functionally determines player $b$ and denote this by $a \triangleright b$.

Note that, in the case of the coordination game, we also have $b \triangleright a$. However, for the game described by Table 2, the statement $a \triangleright b$ is true, but $b \triangleright a$ is false.

The main focus of this article is functional dependence in multiplayer games. For example, consider a "parity" game with three players $a, b, c$. Each of the players picks 0 or 1, and all players are rewarded if the sum of all three numbers is even. This game has four different Nash equilibria: $(0,0,0),(0,1,1),(1,0,1)$, and $(1,1,0)$. It is easy to see that knowledge of any two players' strategies in a Nash equilibrium

Table 1 Coordination game.

\begin{tabular}{|c|c|c|}
\hline & $b_{1}$ & $b_{2}$ \\
\hline$a_{1}$ & 1,1 & 0,0 \\
\hline$a_{2}$ & 0,0 & 1,1 \\
\hline
\end{tabular}

Received March 20, 2013; accepted October 29, 2013

First published online February 25, 2016

2010 Mathematics Subject Classification: Primary 03B80

Keywords: strategic games, functional dependency, axiomatization, completeness

(C) 2016 by University of Notre Dame 10.1215/00294527-3479096 


\title{
Functional Dependence in Strategic Games
}

\author{
Kristine Harjes and Pavel Naumov
}

\begin{abstract}
The article studies properties of functional dependencies between strategies of players in Nash equilibria of multiplayer strategic games. The main focus is on the properties of functional dependencies in the context of a fixed dependency graph for payoff functions. A logical system describing properties of functional dependence for any given graph is proposed and is proven to be complete.
\end{abstract}

\section{Introduction}

Functional dependence. In this article we study dependency between players' strategies in Nash equilibria. For example, the coordination game described by Table 1 has two Nash equilibria: $\left(a_{1}, b_{1}\right)$ and $\left(a_{2}, b_{2}\right)$. Knowing the strategy of player $a$ in a Nash equilibrium of this game, one can predict the strategy of player $b$. We say that player $a$ functionally determines player $b$ and denote this by $a \triangleright b$.

Note that, in the case of the coordination game, we also have $b \triangleright a$. However, for the game described by Table 2, the statement $a \triangleright b$ is true, but $b \triangleright a$ is false.

The main focus of this article is functional dependence in multiplayer games. For example, consider a "parity" game with three players $a, b, c$. Each of the players picks 0 or 1, and all players are rewarded if the sum of all three numbers is even. This game has four different Nash equilibria: $(0,0,0),(0,1,1),(1,0,1)$, and $(1,1,0)$. It is easy to see that knowledge of any two players' strategies in a Nash equilibrium

Table 1 Coordination game.

\begin{tabular}{|c|c|c|}
\hline & $b_{1}$ & $b_{2}$ \\
\hline$a_{1}$ & 1,1 & 0,0 \\
\hline$a_{2}$ & 0,0 & 1,1 \\
\hline
\end{tabular}

Received March 20, 2013; accepted October 29, 2013

First published online February 25, 2016

2010 Mathematics Subject Classification: Primary 03B80

Keywords: strategic games, functional dependency, axiomatization, completeness

(C) 2016 by University of Notre Dame 10.1215/00294527-3479096 


\title{
Functional Dependence in Strategic Games
}

\author{
Kristine Harjes and Pavel Naumov
}

\begin{abstract}
The article studies properties of functional dependencies between strategies of players in Nash equilibria of multiplayer strategic games. The main focus is on the properties of functional dependencies in the context of a fixed dependency graph for payoff functions. A logical system describing properties of functional dependence for any given graph is proposed and is proven to be complete.
\end{abstract}

\section{Introduction}

Functional dependence. In this article we study dependency between players' strategies in Nash equilibria. For example, the coordination game described by Table 1 has two Nash equilibria: $\left(a_{1}, b_{1}\right)$ and $\left(a_{2}, b_{2}\right)$. Knowing the strategy of player $a$ in a Nash equilibrium of this game, one can predict the strategy of player $b$. We say that player $a$ functionally determines player $b$ and denote this by $a \triangleright b$.

Note that, in the case of the coordination game, we also have $b \triangleright a$. However, for the game described by Table 2, the statement $a \triangleright b$ is true, but $b \triangleright a$ is false.

The main focus of this article is functional dependence in multiplayer games. For example, consider a "parity" game with three players $a, b, c$. Each of the players picks 0 or 1, and all players are rewarded if the sum of all three numbers is even. This game has four different Nash equilibria: $(0,0,0),(0,1,1),(1,0,1)$, and $(1,1,0)$. It is easy to see that knowledge of any two players' strategies in a Nash equilibrium

Table 1 Coordination game.

\begin{tabular}{|c|c|c|}
\hline & $b_{1}$ & $b_{2}$ \\
\hline$a_{1}$ & 1,1 & 0,0 \\
\hline$a_{2}$ & 0,0 & 1,1 \\
\hline
\end{tabular}

Received March 20, 2013; accepted October 29, 2013

First published online February 25, 2016

2010 Mathematics Subject Classification: Primary 03B80

Keywords: strategic games, functional dependency, axiomatization, completeness

(C) 2016 by University of Notre Dame 10.1215/00294527-3479096 


\title{
Functional Dependence in Strategic Games
}

\author{
Kristine Harjes and Pavel Naumov
}

\begin{abstract}
The article studies properties of functional dependencies between strategies of players in Nash equilibria of multiplayer strategic games. The main focus is on the properties of functional dependencies in the context of a fixed dependency graph for payoff functions. A logical system describing properties of functional dependence for any given graph is proposed and is proven to be complete.
\end{abstract}

\section{Introduction}

Functional dependence. In this article we study dependency between players' strategies in Nash equilibria. For example, the coordination game described by Table 1 has two Nash equilibria: $\left(a_{1}, b_{1}\right)$ and $\left(a_{2}, b_{2}\right)$. Knowing the strategy of player $a$ in a Nash equilibrium of this game, one can predict the strategy of player $b$. We say that player $a$ functionally determines player $b$ and denote this by $a \triangleright b$.

Note that, in the case of the coordination game, we also have $b \triangleright a$. However, for the game described by Table 2, the statement $a \triangleright b$ is true, but $b \triangleright a$ is false.

The main focus of this article is functional dependence in multiplayer games. For example, consider a "parity" game with three players $a, b, c$. Each of the players picks 0 or 1, and all players are rewarded if the sum of all three numbers is even. This game has four different Nash equilibria: $(0,0,0),(0,1,1),(1,0,1)$, and $(1,1,0)$. It is easy to see that knowledge of any two players' strategies in a Nash equilibrium

Table 1 Coordination game.

\begin{tabular}{|c|c|c|}
\hline & $b_{1}$ & $b_{2}$ \\
\hline$a_{1}$ & 1,1 & 0,0 \\
\hline$a_{2}$ & 0,0 & 1,1 \\
\hline
\end{tabular}

Received March 20, 2013; accepted October 29, 2013

First published online February 25, 2016

2010 Mathematics Subject Classification: Primary 03B80

Keywords: strategic games, functional dependency, axiomatization, completeness

(C) 2016 by University of Notre Dame 10.1215/00294527-3479096 


\title{
Functional Dependence in Strategic Games
}

\author{
Kristine Harjes and Pavel Naumov
}

\begin{abstract}
The article studies properties of functional dependencies between strategies of players in Nash equilibria of multiplayer strategic games. The main focus is on the properties of functional dependencies in the context of a fixed dependency graph for payoff functions. A logical system describing properties of functional dependence for any given graph is proposed and is proven to be complete.
\end{abstract}

\section{Introduction}

Functional dependence. In this article we study dependency between players' strategies in Nash equilibria. For example, the coordination game described by Table 1 has two Nash equilibria: $\left(a_{1}, b_{1}\right)$ and $\left(a_{2}, b_{2}\right)$. Knowing the strategy of player $a$ in a Nash equilibrium of this game, one can predict the strategy of player $b$. We say that player $a$ functionally determines player $b$ and denote this by $a \triangleright b$.

Note that, in the case of the coordination game, we also have $b \triangleright a$. However, for the game described by Table 2, the statement $a \triangleright b$ is true, but $b \triangleright a$ is false.

The main focus of this article is functional dependence in multiplayer games. For example, consider a "parity" game with three players $a, b, c$. Each of the players picks 0 or 1, and all players are rewarded if the sum of all three numbers is even. This game has four different Nash equilibria: $(0,0,0),(0,1,1),(1,0,1)$, and $(1,1,0)$. It is easy to see that knowledge of any two players' strategies in a Nash equilibrium

Table 1 Coordination game.

\begin{tabular}{|c|c|c|}
\hline & $b_{1}$ & $b_{2}$ \\
\hline$a_{1}$ & 1,1 & 0,0 \\
\hline$a_{2}$ & 0,0 & 1,1 \\
\hline
\end{tabular}

Received March 20, 2013; accepted October 29, 2013

First published online February 25, 2016

2010 Mathematics Subject Classification: Primary 03B80

Keywords: strategic games, functional dependency, axiomatization, completeness

(C) 2016 by University of Notre Dame 10.1215/00294527-3479096 


\title{
Functional Dependence in Strategic Games
}

\author{
Kristine Harjes and Pavel Naumov
}

\begin{abstract}
The article studies properties of functional dependencies between strategies of players in Nash equilibria of multiplayer strategic games. The main focus is on the properties of functional dependencies in the context of a fixed dependency graph for payoff functions. A logical system describing properties of functional dependence for any given graph is proposed and is proven to be complete.
\end{abstract}

\section{Introduction}

Functional dependence. In this article we study dependency between players' strategies in Nash equilibria. For example, the coordination game described by Table 1 has two Nash equilibria: $\left(a_{1}, b_{1}\right)$ and $\left(a_{2}, b_{2}\right)$. Knowing the strategy of player $a$ in a Nash equilibrium of this game, one can predict the strategy of player $b$. We say that player $a$ functionally determines player $b$ and denote this by $a \triangleright b$.

Note that, in the case of the coordination game, we also have $b \triangleright a$. However, for the game described by Table 2, the statement $a \triangleright b$ is true, but $b \triangleright a$ is false.

The main focus of this article is functional dependence in multiplayer games. For example, consider a "parity" game with three players $a, b, c$. Each of the players picks 0 or 1, and all players are rewarded if the sum of all three numbers is even. This game has four different Nash equilibria: $(0,0,0),(0,1,1),(1,0,1)$, and $(1,1,0)$. It is easy to see that knowledge of any two players' strategies in a Nash equilibrium

Table 1 Coordination game.

\begin{tabular}{|c|c|c|}
\hline & $b_{1}$ & $b_{2}$ \\
\hline$a_{1}$ & 1,1 & 0,0 \\
\hline$a_{2}$ & 0,0 & 1,1 \\
\hline
\end{tabular}

Received March 20, 2013; accepted October 29, 2013

First published online February 25, 2016

2010 Mathematics Subject Classification: Primary 03B80

Keywords: strategic games, functional dependency, axiomatization, completeness

(C) 2016 by University of Notre Dame 10.1215/00294527-3479096 


\title{
Functional Dependence in Strategic Games
}

\author{
Kristine Harjes and Pavel Naumov
}

\begin{abstract}
The article studies properties of functional dependencies between strategies of players in Nash equilibria of multiplayer strategic games. The main focus is on the properties of functional dependencies in the context of a fixed dependency graph for payoff functions. A logical system describing properties of functional dependence for any given graph is proposed and is proven to be complete.
\end{abstract}

\section{Introduction}

Functional dependence. In this article we study dependency between players' strategies in Nash equilibria. For example, the coordination game described by Table 1 has two Nash equilibria: $\left(a_{1}, b_{1}\right)$ and $\left(a_{2}, b_{2}\right)$. Knowing the strategy of player $a$ in a Nash equilibrium of this game, one can predict the strategy of player $b$. We say that player $a$ functionally determines player $b$ and denote this by $a \triangleright b$.

Note that, in the case of the coordination game, we also have $b \triangleright a$. However, for the game described by Table 2, the statement $a \triangleright b$ is true, but $b \triangleright a$ is false.

The main focus of this article is functional dependence in multiplayer games. For example, consider a "parity" game with three players $a, b, c$. Each of the players picks 0 or 1, and all players are rewarded if the sum of all three numbers is even. This game has four different Nash equilibria: $(0,0,0),(0,1,1),(1,0,1)$, and $(1,1,0)$. It is easy to see that knowledge of any two players' strategies in a Nash equilibrium

Table 1 Coordination game.

\begin{tabular}{|c|c|c|}
\hline & $b_{1}$ & $b_{2}$ \\
\hline$a_{1}$ & 1,1 & 0,0 \\
\hline$a_{2}$ & 0,0 & 1,1 \\
\hline
\end{tabular}

Received March 20, 2013; accepted October 29, 2013

First published online February 25, 2016

2010 Mathematics Subject Classification: Primary 03B80

Keywords: strategic games, functional dependency, axiomatization, completeness

(C) 2016 by University of Notre Dame 10.1215/00294527-3479096 


\title{
Functional Dependence in Strategic Games
}

\author{
Kristine Harjes and Pavel Naumov
}

\begin{abstract}
The article studies properties of functional dependencies between strategies of players in Nash equilibria of multiplayer strategic games. The main focus is on the properties of functional dependencies in the context of a fixed dependency graph for payoff functions. A logical system describing properties of functional dependence for any given graph is proposed and is proven to be complete.
\end{abstract}

\section{Introduction}

Functional dependence. In this article we study dependency between players' strategies in Nash equilibria. For example, the coordination game described by Table 1 has two Nash equilibria: $\left(a_{1}, b_{1}\right)$ and $\left(a_{2}, b_{2}\right)$. Knowing the strategy of player $a$ in a Nash equilibrium of this game, one can predict the strategy of player $b$. We say that player $a$ functionally determines player $b$ and denote this by $a \triangleright b$.

Note that, in the case of the coordination game, we also have $b \triangleright a$. However, for the game described by Table 2, the statement $a \triangleright b$ is true, but $b \triangleright a$ is false.

The main focus of this article is functional dependence in multiplayer games. For example, consider a "parity" game with three players $a, b, c$. Each of the players picks 0 or 1, and all players are rewarded if the sum of all three numbers is even. This game has four different Nash equilibria: $(0,0,0),(0,1,1),(1,0,1)$, and $(1,1,0)$. It is easy to see that knowledge of any two players' strategies in a Nash equilibrium

Table 1 Coordination game.

\begin{tabular}{|c|c|c|}
\hline & $b_{1}$ & $b_{2}$ \\
\hline$a_{1}$ & 1,1 & 0,0 \\
\hline$a_{2}$ & 0,0 & 1,1 \\
\hline
\end{tabular}

Received March 20, 2013; accepted October 29, 2013

First published online February 25, 2016

2010 Mathematics Subject Classification: Primary 03B80

Keywords: strategic games, functional dependency, axiomatization, completeness

(C) 2016 by University of Notre Dame 10.1215/00294527-3479096 


\title{
Functional Dependence in Strategic Games
}

\author{
Kristine Harjes and Pavel Naumov
}

\begin{abstract}
The article studies properties of functional dependencies between strategies of players in Nash equilibria of multiplayer strategic games. The main focus is on the properties of functional dependencies in the context of a fixed dependency graph for payoff functions. A logical system describing properties of functional dependence for any given graph is proposed and is proven to be complete.
\end{abstract}

\section{Introduction}

Functional dependence. In this article we study dependency between players' strategies in Nash equilibria. For example, the coordination game described by Table 1 has two Nash equilibria: $\left(a_{1}, b_{1}\right)$ and $\left(a_{2}, b_{2}\right)$. Knowing the strategy of player $a$ in a Nash equilibrium of this game, one can predict the strategy of player $b$. We say that player $a$ functionally determines player $b$ and denote this by $a \triangleright b$.

Note that, in the case of the coordination game, we also have $b \triangleright a$. However, for the game described by Table 2, the statement $a \triangleright b$ is true, but $b \triangleright a$ is false.

The main focus of this article is functional dependence in multiplayer games. For example, consider a "parity" game with three players $a, b, c$. Each of the players picks 0 or 1, and all players are rewarded if the sum of all three numbers is even. This game has four different Nash equilibria: $(0,0,0),(0,1,1),(1,0,1)$, and $(1,1,0)$. It is easy to see that knowledge of any two players' strategies in a Nash equilibrium

Table 1 Coordination game.

\begin{tabular}{|c|c|c|}
\hline & $b_{1}$ & $b_{2}$ \\
\hline$a_{1}$ & 1,1 & 0,0 \\
\hline$a_{2}$ & 0,0 & 1,1 \\
\hline
\end{tabular}

Received March 20, 2013; accepted October 29, 2013

First published online February 25, 2016

2010 Mathematics Subject Classification: Primary 03B80

Keywords: strategic games, functional dependency, axiomatization, completeness

(C) 2016 by University of Notre Dame 10.1215/00294527-3479096 


\title{
Functional Dependence in Strategic Games
}

\author{
Kristine Harjes and Pavel Naumov
}

\begin{abstract}
The article studies properties of functional dependencies between strategies of players in Nash equilibria of multiplayer strategic games. The main focus is on the properties of functional dependencies in the context of a fixed dependency graph for payoff functions. A logical system describing properties of functional dependence for any given graph is proposed and is proven to be complete.
\end{abstract}

\section{Introduction}

Functional dependence. In this article we study dependency between players' strategies in Nash equilibria. For example, the coordination game described by Table 1 has two Nash equilibria: $\left(a_{1}, b_{1}\right)$ and $\left(a_{2}, b_{2}\right)$. Knowing the strategy of player $a$ in a Nash equilibrium of this game, one can predict the strategy of player $b$. We say that player $a$ functionally determines player $b$ and denote this by $a \triangleright b$.

Note that, in the case of the coordination game, we also have $b \triangleright a$. However, for the game described by Table 2, the statement $a \triangleright b$ is true, but $b \triangleright a$ is false.

The main focus of this article is functional dependence in multiplayer games. For example, consider a "parity" game with three players $a, b, c$. Each of the players picks 0 or 1, and all players are rewarded if the sum of all three numbers is even. This game has four different Nash equilibria: $(0,0,0),(0,1,1),(1,0,1)$, and $(1,1,0)$. It is easy to see that knowledge of any two players' strategies in a Nash equilibrium

Table 1 Coordination game.

\begin{tabular}{|c|c|c|}
\hline & $b_{1}$ & $b_{2}$ \\
\hline$a_{1}$ & 1,1 & 0,0 \\
\hline$a_{2}$ & 0,0 & 1,1 \\
\hline
\end{tabular}

Received March 20, 2013; accepted October 29, 2013

First published online February 25, 2016

2010 Mathematics Subject Classification: Primary 03B80

Keywords: strategic games, functional dependency, axiomatization, completeness

(C) 2016 by University of Notre Dame 10.1215/00294527-3479096 


\title{
Functional Dependence in Strategic Games
}

\author{
Kristine Harjes and Pavel Naumov
}

\begin{abstract}
The article studies properties of functional dependencies between strategies of players in Nash equilibria of multiplayer strategic games. The main focus is on the properties of functional dependencies in the context of a fixed dependency graph for payoff functions. A logical system describing properties of functional dependence for any given graph is proposed and is proven to be complete.
\end{abstract}

\section{Introduction}

Functional dependence. In this article we study dependency between players' strategies in Nash equilibria. For example, the coordination game described by Table 1 has two Nash equilibria: $\left(a_{1}, b_{1}\right)$ and $\left(a_{2}, b_{2}\right)$. Knowing the strategy of player $a$ in a Nash equilibrium of this game, one can predict the strategy of player $b$. We say that player $a$ functionally determines player $b$ and denote this by $a \triangleright b$.

Note that, in the case of the coordination game, we also have $b \triangleright a$. However, for the game described by Table 2, the statement $a \triangleright b$ is true, but $b \triangleright a$ is false.

The main focus of this article is functional dependence in multiplayer games. For example, consider a "parity" game with three players $a, b, c$. Each of the players picks 0 or 1, and all players are rewarded if the sum of all three numbers is even. This game has four different Nash equilibria: $(0,0,0),(0,1,1),(1,0,1)$, and $(1,1,0)$. It is easy to see that knowledge of any two players' strategies in a Nash equilibrium

Table 1 Coordination game.

\begin{tabular}{|c|c|c|}
\hline & $b_{1}$ & $b_{2}$ \\
\hline$a_{1}$ & 1,1 & 0,0 \\
\hline$a_{2}$ & 0,0 & 1,1 \\
\hline
\end{tabular}

Received March 20, 2013; accepted October 29, 2013

First published online February 25, 2016

2010 Mathematics Subject Classification: Primary 03B80

Keywords: strategic games, functional dependency, axiomatization, completeness

(C) 2016 by University of Notre Dame 10.1215/00294527-3479096 